\title{
The year in cardiology: cardiovascular prevention The year in cardiology 2019
}

\author{
"Department of Primary Care and Public Health, Imperial Centre \\ for Cardiovascular Disease Prevention, Imperial College London, \\ London, United Kingdom \\ "Clinic and Polyclinic for Cardiology, University Hospital Leipzig, \\ Liebigstrasse, Leipzig, Germany \\ Unit of Cardiology, Department of Medicine, Karolinska Institute \\ and Karolinska University Hospital, Stockholm, Sweden \\ Department of Clinical Pharmacology, Barts NIHR Biomedical \\ Research Centre, William Harvey Research Institute, London, \\ United Kingdom \\ Department of Cardiology, Charite University Medicine Berlin, \\ Berlin, Germany \\ 'Berlin Institute of Health, German Center of Cardiovascular \\ Research, Partner Site Berlin, Berlin, Germany
}

\section{Address for correspondence:}

Kausik K. Ray

Department of Primary Care and Public Health Imperial Centre for Cardiovascular Disease Prevention

Imperial College London

Reynolds Building

St Dunstan's Road

London W68RP

United Kingdom

Email:

k.ray@imperial.ac.uk

\section{INTRODUCTION}

Advances in genomics, understanding of the effects of cumulative exposure and various environmental risk factors have moved us closer to better models of care focused at early risk assessment and treatment to prevent cardiovascular (CV) disease. We review relevant contributions in 2019 to the field of CV disease prevention, with a focus on epidemiology, lipids, diabetes, and hypertension.

\section{EVOLVING CONCEPTS IN PREVENTION}

Current concepts for risk assessment for the primary prevention of atherosclerotic cardiovascular disease (ASCVD) are based on assessments of multiple risk factors and global risk when one high-risk condition such as diabetes, genetic dyslipidaemia, or hypertension is absent. These are usually measured at a specific time point and predict short-term risk (IOyears) upon which life-long interventions including lifestyle and pharmacotherapy are then based. Advances in genomics may help identify individuals with genetic vulnerability to ASCVD and the recognition of the importance of duration of exposure to risk factors such as low-density lipoprotein (LDL)-cholesterol (LDL-C), blood pressure ${ }^{(1)}$ or number of cigarettes (pack-years) are helping to reshape the paradigm of risk assessment with greater precision (Figure I). These are likely to move the approach of health systems from ones treating disease to ones which aim to preserve health (Figure 2). Central to this aim is the move from short-term risk assessment to lifetime risk and earlier implementation of preventive strategies.(2) In this article, we highlight some of the key scientific observations in the field of prevention in 2019 from risk assessment, and epidemiology with an additional focus on lipids, diabetes, and hypertension.

A recurring observation is that conventional risk assessment is imprecise and the addition of information from imaging consistently helps to correctly reclassify individuals. As a result, the use of imaging and in particular coronary artery calcification (CAC) has been shown to be superior to other modalities and is therefore encouraged among those at intermediate risk and the presence of subclinical atherosclerotic disease supports earlier and more targeted CV prevention strategies in the new ESC/EAS and ESC/EASD 2019 guidelines. ${ }^{(3,4)}$ Moreover, absence of CAC may also reclassify risk down and that should be considered in a shared decision environment. Imaging modalities which lend themselves to machine learning such as evaluation of perivascular fat in cardiac computer tomography may well allow imaging to be scaled up, and become reproducible and cost-effective as part of the risk assessment tool. ${ }^{(5)}$

While imaging is clearly important its use is likely to be useful after decades of exposure to risk factors and still provides assessment for short- to intermediate-term risk. Recently, a lifetime-perspective CardioVascular Disease (LIFE-CVD) model for the estimation of treatment-effects of cholesterol-lowering, blood pressure lowering, antithrombotic therapy, and smoking cessation in apparently healthy people has been developed. This freely accessible online calculator (www.U-Prevent.com) estimates risk and treatment effects in terms of improved 10 year risk, lifetime risk, and life-expectancy free of CVD and is designed to facilitate doctor-patient communication. ${ }^{(6)}$ Large trials of pharmacological intervention assessing outcomes over 
a time horizon of 50 years will never occur. However, the importance of early and sustained reduction in risk factors, notably LDL-C and blood pressure, were highlighted in analyses from UK Biobank where a $1 \mathrm{mmol} / \mathrm{L}$ lower LDL-C and a $10 \mathrm{mmHg}$ lower blood pressure were associated with an $80 \%$ lower risk of CV disease.(I) Put more simply, small differences maintained over a long time produce cumulative benefits. (I) Moreover, higher levels of $\mathrm{CV}$ risk factors are associated with worse brain health across grey and white matter macrostructure and microstructure in relatively healthy middle and older age individuals, suggesting that common risk factor modification could improve a current health burden in late-life, namely dementia. ${ }^{(7)}$

Digital health technology is rapidly advancing and sensors may allow earlier detection of conditions associated with increased CV risk, such as atrial fibrillation (AF). ${ }^{(8)}$ While compelling evidence for their effectiveness is largely lacking, large scale studies have been initiated. The HEARTLIVE study enrolling 150000 participants (>65years of age) is assessing whether earlier detection of AF by a smartwatch sensing technology reduces the risk of $\mathrm{CV}$ events. However, there are also concerns that widespread use of such approaches, particularly in the low risk, younger populations using such devices, may lead to unnecessary medical consultations, ${ }^{\left({ }^{9}\right)}$ making an assessment of studies such as HEARTLIVE in appropriate populations important.

\section{BEHAVIOUR AND ENVIRONMENTAL FACTORS}

\section{Genetics}

Considerable amounts of data have emerged from UK Biobank. However, data are needed on non-European populations as 10 000 of the 500000 cohort are from south Asian or Afrocarribean ancestry.

Behaviour may, in part, have a genetic basis. In a Mendelian randomisation analysis from UK Biobank, genetic variants known to affect educational attainment were associated with health-conscious lifestyle later in life, and which in turn may subsequently affect the risk of coronary artery disease. ${ }^{(10)}$

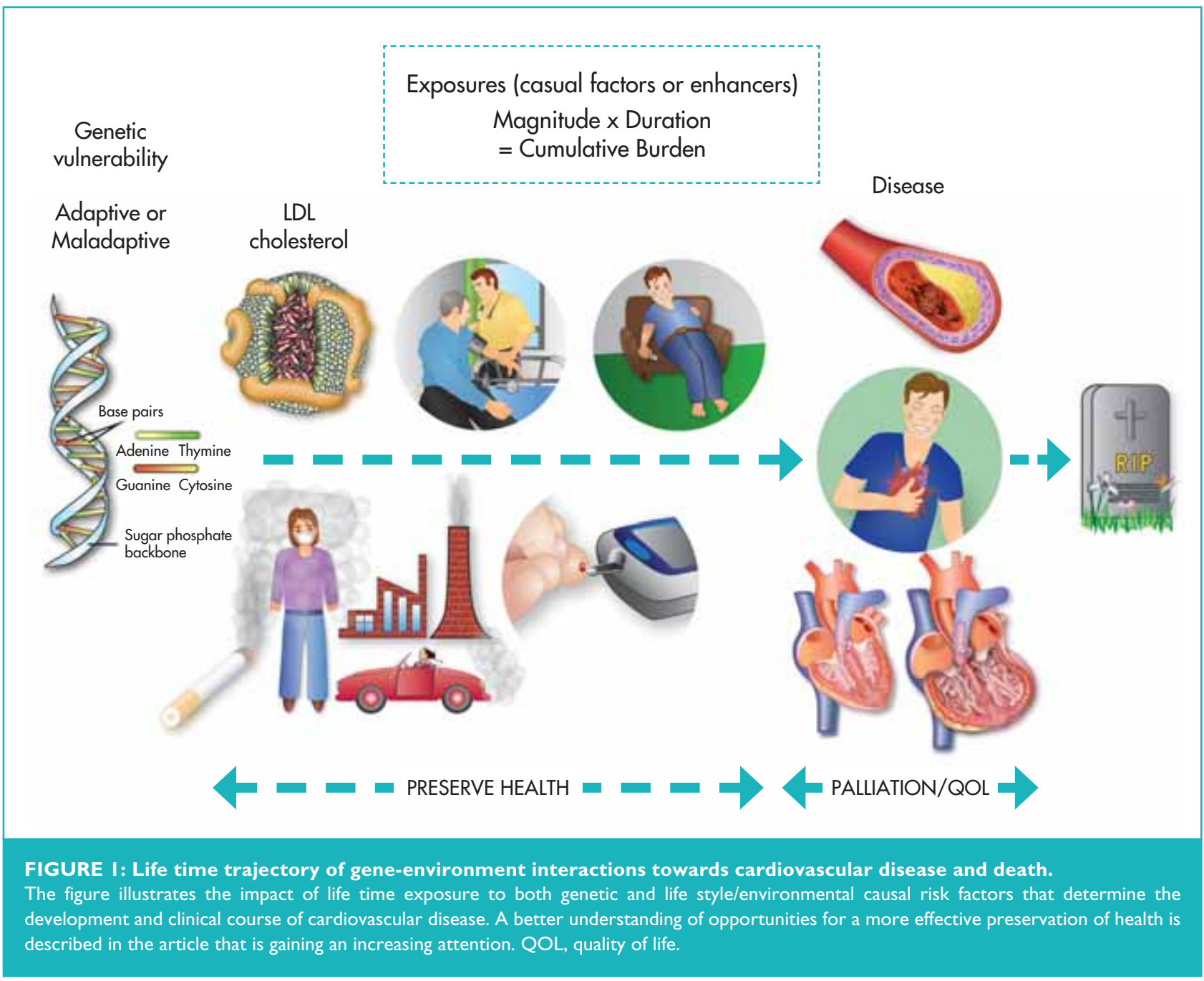




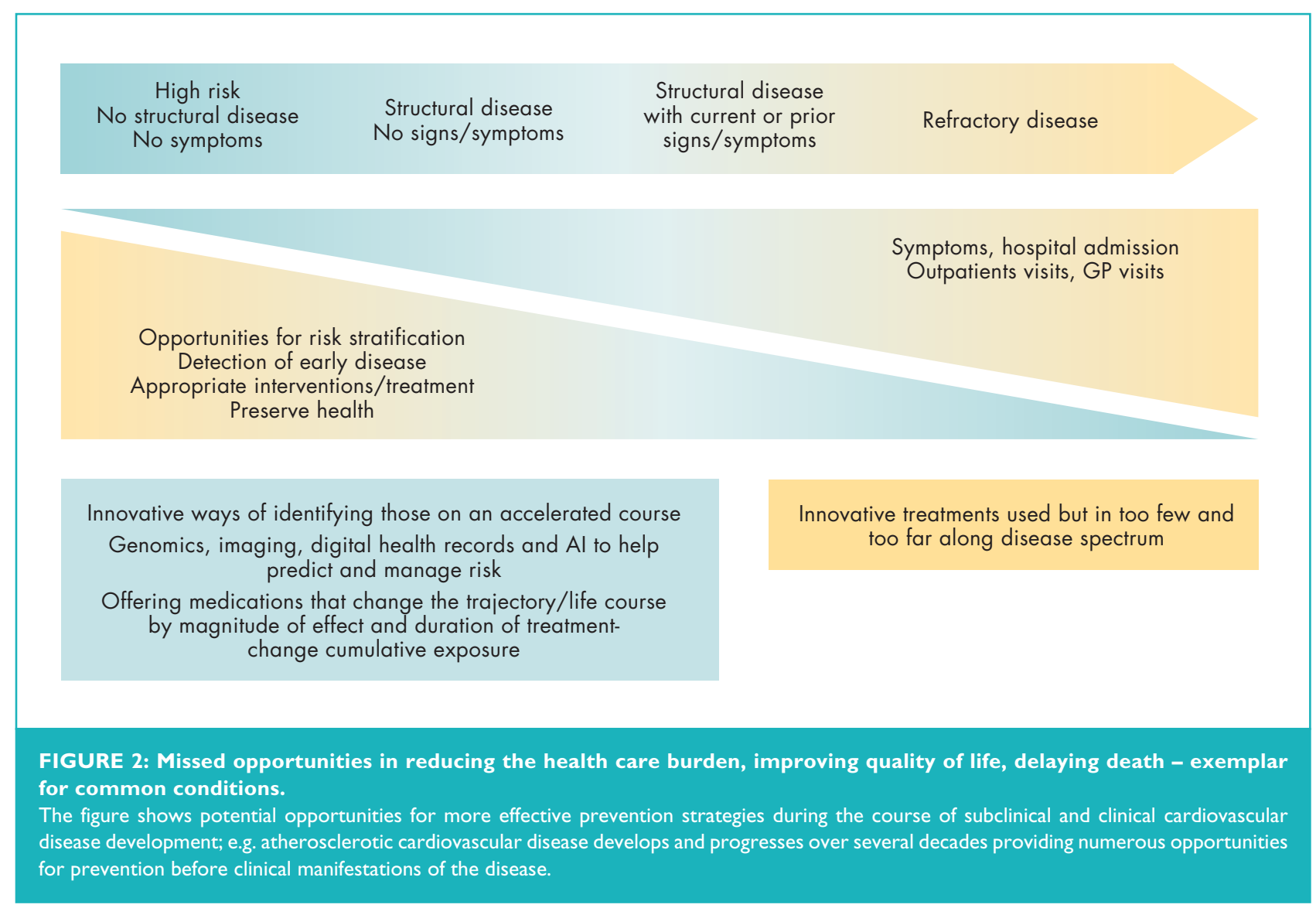

\section{Nutrition}

\section{Red meat}

Data are conflicting with different recommendations regarding red meat consumption. Observational studies suggested potential carcinogenic effects of processed meat.(II) Four systematic reviews on the health effects of red meat and one systematic review on individual health-related values and preferences regarding meat consumption have been published, ${ }^{(12)}$ where the magnitudes of any effect were small. Additionally, these studies report only very low to low certainty for any association of unprocessed or processed red meat intake with CV mortality, diabetes, or cancer. The authors conclude that individuals continue their current consumption of both processed and unprocessed meat, albeit with a weak recommendation because of the low certainty around the evidence. ${ }^{(12)}$ Of note, a recent randomised dietary study suggests that chronic dietary red meat consumption increases systemic levels of trimethylamine $\mathrm{N}$-oxide (TMAO), a microbiome-dependent metabolite, that has been associated with increased CV risk, (13) but larger studies are needed.

\section{Carbohydrates}

Conflicting data on the role of carbohydrates for ASCVD risk have led to different recommendations. For example, the large
Prospective Urban Rural Epidemiology (PURE) study reported that high carbohydrate intake was associated with higher risk of total mortality.(14) In contrast, a recent analysis of the National Health and Nutrition Examination Survey (NHANES; 1999 - 2010) suggests exactly the opposite, with low carbohydrate diets associated with excess overall and cause-specific mortality. ${ }^{(15)}$ Nutritional epidemiology carries the risk of confounding by social and economic factors. The underlying causal association (if any) of behaviour, such as "skipping breakfast", with ASCVD may be unrelated to discussions about the benefit of fat vs. carbohydrates, ${ }^{(16)}$ therefore, the evidence to support population-level interventions such as increasing the price of high sugar snacks appears incomplete, especially since this would differentially affect low-income individuals. (17) More recently, the totality of the literature of this topic was summarised by a $U$-shaped relationship between carbohydrate intake and mortality. ${ }^{(18)}$ The authors conclude that "taking all the studies into account, the message of moderation is perhaps the most convincing one of all - diets that focus too heavily on a single macronutrient, whether extreme protein, carbohydrate, or fat intake, may adversely impact health. The best advice seems to be to select whole foods from a variety of sources and avoid dietary extremism. For now, for carbohydrates, everything in moderation seems to carry the day".(18) 


\section{Body weight}

The notion that the effect of any diet on body weight is in turn proportional to risk of ASCVD may be over-simplistic. (19) In the Women's Health Initiative, during a median of 17.9 years of follow-up, whole body fat mass was not associated with incident ASCVD among normal weight post-menopausal women. Interestingly, the distribution of fat was, with higher trunk fat associated with higher risk of ASCVD, while higher leg fat predicted lower risk. ${ }^{(2)}$ These data suggest an adverse fat distribution and risk can be characterised by increased (unfavourable) abdominal/visceral (trunk) and decreased (beneficial) lower body (leg) fat that is independent of body fat mass. Future research should address potential mechanisms for the development of adverse fat distribution and how it may be linked to atherosclerosis. ${ }^{(19,20)}$

\section{Sleep duration}

Data from the Prospective Urban Rural Epidemiology (PURE) study on 116632 with follow-up of 7.8 years that show that estimated total sleep duration of 6 - 8 hours per day is associated with the lowest risk of deaths and major CV events. ${ }^{(21)}$ Interestingly, a neuro-immune axis that links sleep to haematopoiesis and atherosclerosis has been identified and provides a mechanistic rationale for disturbed sleep and increased CV risk. (22)

\section{Smoking}

Recent data from the Framingham Heart Study provide quantitative information on the positive health effects of smoking cessation based on $>25$ years of follow-up showing that quitting within 5 years was associated with $39 \%$ lower risk of incident CVD compared with current smokers. Also, among heavy smokers, smoking cessation was associated with lower risk of CVD relative to current smokers. ${ }^{(23)}$ The health effects of e-cigarettes (so-called "vaping") are still uncertain, and recent case reports suggest potential emerging clinical syndromes that are not yet completely understood. ${ }^{(24)}$

\section{Exercise}

Increased physical activity, at any intensity and less time spent sedentary, is associated with substantially reduced risk for premature mortality. (25) However, translation into patient care and individualised training recommendations remain a challenge. A randomised controlled trial showed that endurance and interval training but not resistance training, induced effects on circulating blood cells that are important for cellular senescence and regenerative capacity, showing that different training modalities exert differential cellular and vascular effects contributing to vascular health. (26)

\section{Noise, pollution, and workplace}

There is increasing awareness of associations between our environment and health. For instance, ambient air pollution has been linked to an excess annual mortality rate of 659000 in the European Union (EU-28), with the majority attributable to $\mathrm{CV}$ causes. ${ }^{(27)}$ Estimates put attributable per capita annual mortality rate in Europe at 133/100 000, but considerable uncertainty around this estimate remains. ${ }^{(27)}$ In this regard, a nationwide cohort study from Switzerland modelled long-term exposure to noise levels as well as environmental pollutants for each address of four million adults. ${ }^{(28)}$ The data suggest that road traffic, aircraft, and railway noise are each associated with excess mortality from myocardial infarction (MI), independent of air pollution. The authors suggest that air pollution studies not adequately adjusting for noise exposure may overestimate the attributable burden of risk from air pollution. . 28,29$)^{2}$

Finally, large cohort studies from Sweden and Denmark reveal that $9 \%$ reported being bullied at work and 13\% recorded exposure to workplace violence during the preceding year. After adjustment, being bullied at work was associated with $59 \%$ increased risk of ASCVD. The population attributable risk was dose-dependent and overall 5.0\% for workplace bullying and $3.1 \%$ for workplace violence. ${ }^{(30)}$

\section{DYSLIPIDAEMIA AND LIPIDS}

Several clinical trial programmes have studied novel treatment options for modification of lipoprotein-related risk of ASCVD that are described below, e.g. new options for lowering LDLcholesterol and triglyceride-rich lipoproteins. These novel therapeutic approaches will allow a more effective and targeted strategy for management of lipoprotein-related risk in the future (Figure 3).

\section{Low-density lipoprotein-cholesterol}

ATP citrate lyase is an enzyme in the cholesterol-biosynthesis pathway upstream of 3-hydroxy-3-methylglutaryl-coenzyme A reductase (HMGCR), the target of statins. Genetic variants that mimic the effect of ATP citrate lyase inhibitors and statins appeared to lower plasma LDL-cholesterol levels by the same mechanism of action and were associated with similar effects on the risk of $\mathrm{CV}$ disease per unit decrease in the LDLcholesterol level.(31) Bempedoic acid, an inhibitor of ATP citrate lyase, reduced levels of LDL cholesterol by $16.5 \%$ when added to maximally tolerated statin therapy, ${ }^{(32)}$ and a clinical outcomes study is ongoing.

Recent data from trials of ezetemibe and PCSK9 monoclonal antibodies demonstrating consistent evidence of benefit with the achievement of lower risk among patients with lower LDL-C levels have now been incorporated into the new ESC/ EAS treatment guidelines in 2019 , with $55 \mathrm{mg} / \mathrm{dL}$ the new goal for very high-risk patients. (3) 


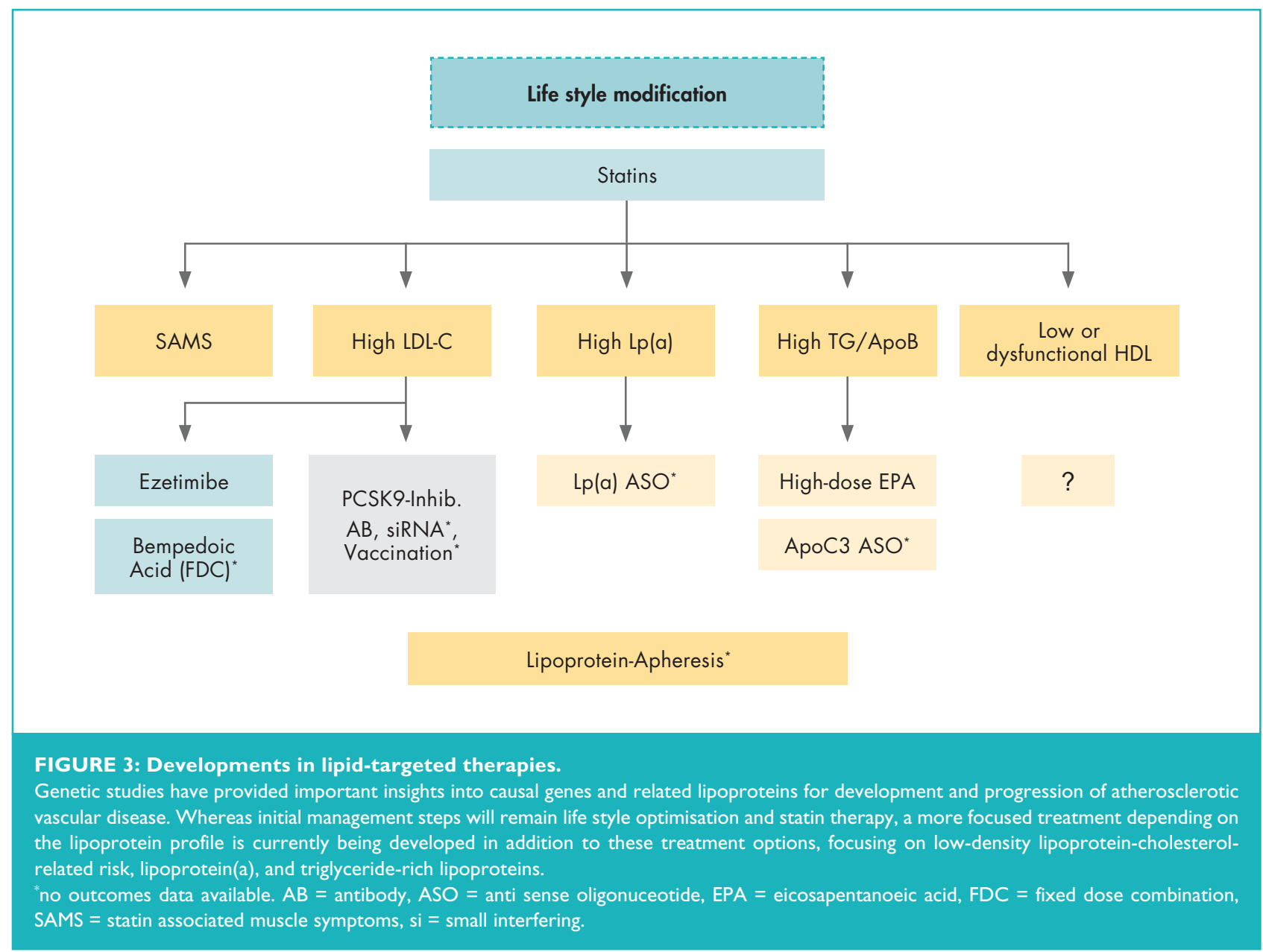

\section{Triglyceride-rich lipoproteins}

In a genetic study, it was observed that triglyceride-lowering lipoprotein lipase variants and LDL-C-lowering LDL-receptor variants were associated with a similar lower risk of coronary heart disease per unit difference in ApoB, suggesting that the clinical benefit of lipid lowering per se is proportional to the absolute change in ApoB. ${ }^{(33)}$ Icosapent ethyl, a highly purified eicosapentaenoic acid ethyl ester, lowered triglyceride levels, and reduced ischaemic events by $26 \%$ in the recent REDUCEIT trial in patients with elevated triglyceride levels compared to mineral oil.(34) The magnitude of benefit was greater than that expected by $A p o B$ changes alone, suggesting mechanisms beyond ApoB lowering. ${ }^{(34,35)}$

\section{Lipoprotein (a)}

A recent analysis of $>65000$ subjects suggested that lipoprotein(a) levels $>93 \mathrm{mg} / \mathrm{dL}$ ( $199 \mathrm{nmol} / \mathrm{L} ; 96 \mathrm{th}$ - 100th percentiles) vs. $<10 \mathrm{mg} / \mathrm{dL}$ ( $18 \mathrm{nmol} / \mathrm{L}$; I st - 50th percentiles) was associated with a $50 \%$ excess risk for CV mortality and of $20 \%$ for allcause mortality. ${ }^{(36)}$ The authors hypothesise that elevated lipoprotein(a), (through corresponding low LPA KIV-2 number of repeats) rather than through $L p(a)$ cholesterol content, were the drivers of this excess risk. (36)

\section{HYPERTENSION}

\section{Epidemiology}

Hypertension is a very important risk factor for CV disease and five decades of trials have demonstrated the benefits of pharmacotherapy in reducing CV morbidity and mortality. However, contemporary data reinforce the need for improvement in hypertension healthcare globally. In 12 high-income countries, data from more than half a million participants indicated greatly improved hypertension awareness, treatment, and control since the 1980s, but substantial variations in hypertension prevalence and treatment across countries. ${ }^{(37)}$ Control rates have plateaued in recent decades with rates of treatment coverage $\sim 80 \%$ and control $\sim 70 \%$ in best performing countries. Conversely, in 44 low-income and middle-income countries, only $40 \%$ of those with hypertension were diagnosed, with 30\% receiving antihypertensive medication, and 10\% controlled with disparity across countries and sub-Saharan Africa performing the worst. ${ }^{(38)}$

\section{Blood pressure measurement}

A study from 1.3 million North American patients has shown that both systolic blood pressure (SBP) and diastolic blood 
pressure (DBP) independently predicted $\mathrm{MI}$, ischaemic/haemorrhagic stroke with a greater effect of systolic hypertension. ${ }^{(39)}$ Importantly, the relationship between SBP, DBP, and events is independent of treatment threshold $(\geq 140 / 90 \mathrm{mmHg}$ vs. $\geq 130 / 80 \mathrm{mmHg}$ ), supporting the more proactive management of hypertension in high-risk individuals in recent guidelines. ${ }^{(40-42)}$ The IDACO investigators observed in a study of I I 35 adults, that higher 24 hours and night-time SBP were significantly associated with greater risks of death and CV events even after adjusting for other office-based or ambulatory $\mathrm{BP}$ measurements, ${ }^{(43)}$ reinforcing recent guidelines recommending the routine use of ambulatory BP monitoring (ABPM) for BP assessment.

\section{Treatment}

\section{Pharmacotherapy}

While there have been no developments in novel therapies for hypertension, an increasing focus is the use of multidrug combinations, even as an initial step in treatment. Most recently, the WHO added fixed dose combination antihypertensive medications to the Essential Medicines List, with the aim of addressing inequalities in treatment and control in low to middle income countries (LMIC). ${ }^{(44)}$ This approach has already demonstrated cost-effectiveness in patients in Sri Lanka with mild-moderate hypertension treated with a triple pill strategy vs. usual care, providing the first economic evaluation of a triple-pill approach. ${ }^{(45)}$

Evidence is accumulating to support nocturnal dosing of antihypertensive medication, with the Hygia Chronotherapy trial, the first ABPM-based outcome study providing evidence that bed-time dosing of $\geq 1$ antihypertensive drug, vs. morning, results in better ambulatory BP control, lower sleep-time BP and improved nocturnal dipper status. ${ }^{(46)}$ Despite modest differences in $\mathrm{BP},{ }^{(46)}$ there was a disproportionate reduction in CVD morbidity and mortality with bedtime dosing with no safety signal noted. Whether this is real requires independent confirmation.

\section{Device therapy}

Endovascular renal denervation (RDN) aims to achieve durable hypertension control through interruption of renal sympathetic nervous system signalling. The open label, single arm Global Symplicity Registry have reported significant and sustained reductions in ambulatory and office BP $(-16.5 \pm 28.6 \mathrm{mmHg}$ and $-8.0 \pm 20.0 \mathrm{mmHg}$, respectively) 3 years post-radiofrequency ablation with no safety signal and preserved renal function in 1742 patients. ${ }^{(47)}$ Furthermore, the RADIANCEHTN SOLO investigators have now shown that the effects of endovascular ultrasound RDN in patients with mildmoderate hypertension are preserved at 6 months, with less medication burden compared with sham control. ${ }^{(13,48)}$ It is unclear which, if any, of the technologies to achieve RDN is superior: radiofrequency (RF) vs. ultrasound (US) vs. alcohol chemical ablation. However, the RADIOSOUND-HTN investigators have shown in patients with resistant hypertension, endovascular US-based RDN achieved similar BP reduction to RF ablation of the main arteries, accessories, and side branches, but was superior to RF ablation of the main renal arteries only. ${ }^{(49)}$ Furthermore, while the search for marker of procedural success and predictors of response to RDN is ongoing, the SPYRAL HTN-OFF MED investigators have demonstrated that RF RDN in patients with mild-moderate hypertension resulted in significant heart rate reduction compared to sham and that hypertensive patients with higher heart rates may be more likely to respond. ${ }^{(48)}$

\section{DIABETES}

The prevalence of diabetes is increasing, with $>425$ million already affected globally, potentially growing to 629 million by 2045. ${ }^{(4)}$ As diabetes doubles the risk of CVD, the increase in prevalence will increase the population attributable risk disproportionately in low-middle income countries where the disposable income and economic growth coupled with sedentary lifestyle are seeing the greatest rise in diabetes prevalence. Novel therapeutic options now offer a chance to move away from prior glucose-centric approaches in diabetes care to those aimed at preventing cardio-renal complications, as evidenced by the 2019 ESC guidelines on diabetes, prediabetes, and CV diseases developed in collaboration with the European Association for the Study of Diabetes (EASD).(4) A key premise of these is the classification of absolute CV risk as the first step, into Very high, High, and Moderate risk. Based on the results of recent trials, using both GLPIRAs and SGLT2 inhibitors, in the 2019 guidelines, these drug classes are recommended as first-line therapy in patients with T2DM and established ASCVD or at high/very high CV risk, such as those with target-organ damage or multiple risk factors instead of metformin. ${ }^{(4)}$ Among those already on metformin, GLPI-RAs and SGLT2 inhibitors should be added for $\mathrm{CV}$ risk reduction with the aim of moving away from a $\mathrm{HbA}$ Ic-centric approach to one which prevents CV disease.

Notable contributions from several large trials in 2019 include the REWIND trial(50) assessing the effect of once weekly subcutaneous dulaglutide vs. placebo on three-point major adverse cardiac events (MACE) in 9901 patients with T2DM, who had either a previous CV event or multiple risk factors. Over 5.4 years of follow-up, the primary composite outcome occurred in $12.0 \%$ of participants in the dulaglutide group and in $13.4 \%$ in the placebo group, reflecting a significant 12\% relative risk reduction. The DECLARE-TIMI 58 trial(51) investigated the effect of dapagliflozin vs. placebo in 17160 patients with DM and established CVD or multiple risk factors. After 
4.2 years of follow-up, the pre-specified criterion for noninferiority for the composite MACE was met by dapagliflozin compared with placebo. In 2 primary efficacy analyses, dapagliflozin did not significantly reduce 3P-MACE, but resulted in a lower rate of the combined endpoint of $\mathrm{CV}$ death or $\mathrm{HF}$ hospitalisation by $17 \%$ (4.9\% vs. 5.8\% absolute difference). The benefit on heart failure was similar in patients with CVD as well as those with multiple risk factors only. A recent metaanalysis of the SGLT2i trials suggested consistent benefits on reducing the composite of $\mathrm{HF}$ hospitalisation or $\mathrm{CV}$ death, as well as on the progression of kidney disease, regardless of presence of established CVD, while the reduction in MACE was only apparent in ASCVD patients. ${ }^{(52)}$ Previous CVOTs with SGLT2 inhibitors demonstrated renal benefit as a secondary endpoint, but the CREDENCE tria|(53) was the first dedicated study assessing renal preservation with SGLT2i in chronic kidney disease and diabetes (estimated glomerular filtration rate 30 - $<90 \mathrm{~mL} / \mathrm{min} / 1.73 \mathrm{~m}^{2}$ ). Individuals randomised to canagliflozin had a relative reduction in the primary renal outcome of $30 \%$ compared to placebo. In addition, canagliflozin significantly reduced the prespecified secondary CV outcomes of 3P-MACE by $20 \%$ and hospitalisation for heart failure by $29 \%$ compared with placebo. More recently, there is now compelling evidence that SGLT2 inhibition reduces heart failure in populations with heart failure and reduced ejection fraction equally among those with or without diabetes in the DAPA CHF trial. ${ }^{(54)}$

\section{INFLAMMATION AND THROMBOSIS}

The CANTOS trial provided the first evidence that targeting inflammation reduced CV outcomes in those with established disease. Ultimately cost, questions regarding duration of therapy and efficacy vs. safety "trade off " with increased infections have not seen the development of IL-I beta antagonism. Targeting inflammation indirectly, with low-cost safe alternatives has been sought with methotrexate showing no benefit. Among patients with a recent $\mathrm{MI}$, low-dose colchicine reduced a broad composite CV endpoint including revascularisation by 23\% (1.6\% absolute benefit) in the COLCOT trial.(55) Colchicine use was associated with an absolute excess of $0.8 \%$ in diarrhoea (NS) and $0.5 \%$ in pneumonia $(P=0.03)$.

Finally, aspirin clearly has net benefit (more CV events voided than significant bleeds caused) in the setting of established CV disease or secondary prevention. However, the observation that in over 100000 patients in primary prevention trials of aspirin demonstrated an excess of about 2.5 excess major bleeds for each non-fatal $\mathrm{Ml}$ averted and no mortality benefit over 5 years. ${ }^{(56)}$ As such, aspirin is not routinely recommended in the ESC guidelines in the setting of primary prevention. ${ }^{(57)}$

\section{SUMMARY AND CONCLUSIONS}

The present article summarises important advances in the field of CV prevention in 2019. We have highlighted the increasing role of considering lifetime $\mathrm{CV}$ risk for maintaining $\mathrm{CV}$ health, as well as the need for risk assessment in patients with established ASCVD or diabetes, for which novel and more targeted preventive therapies have been developed and proven effective.

\section{ACKNOWLEDGEMENTS}

The authors are grateful to Mrs Jennifer Landmann for her editorial support (Imperial College London). K.R. acknowledges support of the NIHR Imperial Biomedical Research Centre. Imperial College London is grateful for support from the NW London NIHR Applied Research Collaboration. The views expressed in this publication are those of the authors and not necessarily those of the NIHR or the Department of Health and Social Care. U.L. acknowledges support from the Leducq Foundation, Berlin Institute of Health, and German Center of Cardiovascular Research (DZHK).

Conflict of interest: K.R. reports personal fees from The Medicines Company Aegerion, Astra Zeneca, Cerenis, Akcea, Medicines Company, Kowa, Novartis, Cipla, Lilly, Algorithm, Takeda, Boehringer Ingelheim, Abbvie, Silence Therapeutics, Dr Reddys, Bayer, Daiichi Sankyo, Esperion, Abbvie, Zuelling Pharma, Resverlogix, outside the submitted work; grants and personal fees from Amgen, Sanofi/Regeneron, Pfizer, MSD. F.C. reports personal fees from AstraZeneca, Boehringer Ingelheim, Bristol Myers Squibb, Lilly, Merck, Mundipharma, Novo Nordisk, Pfizer; grants from the Swedish Research Council VR 201602706, Swedish Heart and Lung Foundation 20170717, and Konung Gustav: Vs och Drottning Victorias Frimurarestiftelse. M.D.L. is funded by the Barts Charity and is a consultant to Medtronic, Ablative Solutions, ReCor Medical, Vascular Dynamics, ROX Medical, and Tarilian Laser Technologies and has received speaker fees from CVRx. U.L. reports personal lecture and advisory fees from The Medicines Company, Bayer, Sanofi, Amgen, Berlin Chemie, Abbott, Boston Scientific, and Novartis. U.L. reports personal lecture or advisory fees from Amgen, Bayer, Boehringer, Sanofi, Amgen, and Novartis. 
I. Ference BA, Bhatt DL, Catapano AL, et al. Association of genetic variants related to combined exposure to lower low-density lipoproteins and lower systolic blood pressure with lifetime risk of cardiovascular disease. JAMA 2019;322:1381.

2. Leistner DM, Landmesser U. Maintaining cardiovascular health in the digital era. Eur Heart J 2019;40:9-12.

3. Mach F, Baigent C, Catapano AL, et al.; ESC Scientific Document Group. 2019 ESC/EAS Guidelines for the management of dyslipidaemias: Lipid modification to reduce cardiovascular risk. Eur Heart J 2019;doi:10.1093/ eurheartj/ehz455.

4. Cosentino F, Grant PJ, Aboyans V, et al.; ESC Scientific Document Group. 2019 ESC Guidelines on diabetes, pre-diabetes, and cardiovascular diseases developed in collaboration with the EASD. Eur Heart J 2019;doi: 10.1093/ eurheartj/ehz486.

5. Oikonomou EK, Williams MC, Kotanidis CP, et al. A novel machine learningderived radiotranscriptomic signature of perivascular fat improves cardiac risk prediction using coronary CT angiography. Eur Heart J 2019; doi: 10.1093/eurheartj/ehz592.

6. Jaspers NEM, Blaha MJ, Matsushita K, et al. Prediction of individualised lifetime benefit from cholesterol lowering, blood pressure lowering, antithrombotic therapy, and smoking cessation in apparently healthy people. Eur Heart J 2019;doi: 10.1093/eurheartj/ehz239.

7. Cox SR, Lyall DM, Ritchie SJ, et al. Associations between vascular risk factors and brain MRI indices in UK Biobank. Eur Heart J 2019:40:2290-2300.

8. Sim I. Mobile devices and health. N Engl J Med 2019;381:956-968.

9. Topol Ej. High-performance medicine: The convergence of human and artificial intelligence. Nat Med 2019;25:44-56.

10. Zeng L, Ntalla I, Kessler T, et al. Genetically modulated educational attainment and coronary disease risk. Eur Heart J 2019;40:2413-2420.

II. Bouvard V, Loomis D, Guyton KZ, et al. Carcinogenicity of consumption of red and processed meat. Lancet Oncol 2015;16:1599-1600.

12. Johnston BC, Zeraatkar D, Han MA, et al. Unprocessed red meat and processed meat consumption: Dietary guideline recommendations from the Nutritional Recommendations (NutriRECS) Consortium. Ann Intern Med 2019;171:756.

13. Azizi M, Schmieder RE, Mahfoud F, et al.; RADIANCE-HTN Investigators. Six-month results of treatment-blinded medication titration for hypertension control following randomisation to endovascular ultrasound renal denervation or a Sham procedure in the RADIANCE-HTN SOLO trial. Circulation 2019; 139:2542.

14. Dehghan M, Mente A, Zhang X, et al.; Prospective Urban Rural Epidemiology (PURE) study investigators. Associations of fats and carbohydrate intake with cardiovascular disease and mortality in 18 countries from five continents (PURE): A prospective cohort study. Lancet 2017;390:2050-2062.

15. Mazidi M, Katsiki N, Mikhailidis DP, et al. Lower carbohydrate diets and allcause and cause-specific mortality: A population-based cohort study and pooling of prospective studies. Eur Heart J 2019;40:2870-2879.

16. Rong S, Snetselaar LG, Xu G, et al. Association of skipping breakfast with cardiovascular and all-cause mortality. J Am Coll Cardiol 2019;73:2025-2032.

17. Scheelbeek PFD, Cornelsen L, Marteau TM, et al. Potential impact on prevalence of obesity in the UK of a $20 \%$ price increase in high sugar snacks: Modelling study. BMJ 2019;366:14786.

18. De Souza RJ, Dehghan M, Anand SS. Low carb or high carb? Everything in moderation ... until further notice. Eur Heart J 2019;40:2880-2882.

19. Bluher M, Laufs U. New concepts for body shape-related cardiovascular risk: Role of fat distribution and adipose tissue function. Eur Heart J 2019;40: 2856-2858.

20. Chen G-C, Arthur R, lyengar NM, et al. Association between regional body fat and cardiovascular disease risk among postmenopausal women with normal body mass index. Eur Heart J 2019;40:2849-2855.

21. Wang C, Bangdiwala SI, Rangarajan S, et al. Association of estimated sleep duration and naps with mortality and cardiovascular events: A study of I I6 632 people from 2 I countries. Eur Heart J 2019;40: 1620- 1629.
22. McAlpine CS, Kiss MG, Rattik S, et al. Sleep modulates haematopoiesis and protects against atherosclerosis. Nature 2019;566:383-387.

23. Duncan MS, Freiberg MS, Greevy RAJr, et al. Association of smoking cessation with subsequent risk of cardiovascular disease. JAMA 2019;322: 642-650.

24. Layden JE, Ghinai I, Pray I, et al. Pulmonary illness related to e-cigarette use in Illinois and Wisconsin - preliminary report. N Engl J Med 2020; 382(10):903-916.

25. Ekelund U, Tarp J, Steene-Johannessen J, et al. Dose-response associations between accelerometry measured physical activity and sedentary time and all cause mortality: Systematic review and harmonised meta-analysis. BMJ 2019;366:14570

26. Werner CM, Hecksteden A, Morsch A, et al. Differential effects of endurance, interval, and resistance training on telomerase activity and telomere length in a randomised, controlled study. Eur Heart J 2019;40:34-46.

27. Lelieveld J, Klingmüller K, Pozzer A, et al. Cardiovascular disease burden from ambient air pollution in Europe reassessed using novel hazard ratio functions. Eur Heart J 2019;40:1590-1596.

28. Héritier $\mathrm{H}$, Vienneau $\mathrm{D}$, Foraster $\mathrm{M}$, et al. A systematic analysis of mutual effects of transportation noise and air pollution exposure on myocardial infarction mortality: A nationwide cohort study in Switzerland. Eur Heart J 2019;40:598-603.

29. Sorensen M, Pershagen G. Transportation noise linked to cardiovascular disease independent from air pollution. Eur Heart J 2019;40:604-606.

30. Xu T, Magnusson Hanson LL, Lange $T$, et al. Workplace bullying and workplace violence as risk factors for cardiovascular disease: A multi-cohort study. Eur Heart I 2019;40: I | 24- I 134

31. Ference BA, Ray KK, Catapano AL, et al. Mendelian randomisation study of ACLY and cardiovascular disease. N Engl J Med 2019;380: I033-1042.

32. Ray KK, Bays HE, Catapano AL, et al. Safety and efficacy of bempedoic acid to reduce LDL cholesterol. N Engl J Med 2019;380:1022-1032

33. Ference BA, Kastelein JJP, Ray KK, et al. Association of triglyceride-lowering $L P L$ variants and $L D L-C$-lowering $L D L R$ variants with risk of coronary heart disease. JAMA 2019:321:364-373.

34. Bhatt DL, Steg PG, Miller M, et al. Cardiovascular risk reduction with icosapent ethyl for hypertriglyceridemia. N Engl J Med 20 19;380: I I-22.

35. Laufs $U$, Parhofer KG, Ginsberg HN, et al. Clinical review on triglycerides. Eur Heart J 2019;doi: 10.1093/eurheartj/ehz785.

36. Langsted A, Kamstrup PR, Nordestgaard BG. High lipoprotein(a) and high risk of mortality. Eur Heart J 2019;40:2760-2770.

37. NCD Risk Factor Collaboration (NCD-RisC). Long-term and recent trends in hypertension awareness, treatment, and control in 12 high-income countries: An analysis of 123 nationally representative surveys. Lancet 2019:394:639-65।.

38. Geldsetzer P, Manne-Goehler J, Marcus M-E, et al. The state of hypertension care in 44 low-income and middle-income countries: A cross-sectional study of nationally representative individual-level data from I.I million adults. Lancet 2019;394:652-662.

39. Flint $A C$, Conell $C$, Ren $X$, et al. Effect of systolic and diastolic blood pressure on cardiovascular outcomes. N Engl J Med 2019;381:243-251.

40. Whelton PK, Carey RM, Aronow WS, et al. 2017 ACC/AHA/AAPA/ABC/ ACPM/AGS/APhA/ASH/ASPC/NMA/PCNA Guideline for the prevention, detection, evaluation, and management of high blood pressure in adults: A report of the American College of Cardiology/American Heart Association Task Force on Clinical Practice Guidelines. I Am Coll Cardiol 2018;71:el27-e248.

41. Williams B, Mancia G, Spiering W, et al.; ESC Scientific Document Group. 2018 ESC/ESH Guidelines for the management of arterial hypertension. Eur Heart J 2018;313:603-698.

42. Williams B, Mancia G, Spiering W, et al.; ESC Scientific Document Group. 2018 ESC/ESH Guidelines for the management of arterial hypertension. Eur Heart J 2018:39:3021-3104

43. Yang W-Y, Melgarejo JD, Thijs L, et al. Association of office and ambulatory blood pressure with mortality and cardiovascular outcomes. JAMA 2019; 322:409-420. 


\section{REFERENCES}

44. Benjamin IJ, Kreutz R, Olsen $\mathrm{MH}$, et al. Fixed-dose combination antihypertensive medications. Lancet 2019;394:637-638.

45. Lung $\mathrm{T}$, Jan $\mathrm{S}$, de Silva HA, et al. Fixed-combination, low-dose, triple-pill antihypertensive medication versus usual care in patients with mild-tomoderate hypertension in Sri Lanka: A within-trial and modelled economic evaluation of the TRIUMPH trial. Lancet Glob Health 2019;7:el 359-el 366.

46. Hermida RC, Crespo JJ, Domínguez-Sardiña M, et al.; Hygia Project Investigators. Bedtime hypertension treatment improves cardiovascular risk reduction: The Hygia Chronotherapy Trial. Eur Heart J 2019;doi: 10.1093/ eurheartj/ehz754.

47. Mahfoud F, Böhm M, Schmieder R, et al. Effects of renal denervation on kidney function and long-term outcomes: 3-year follow-up from the Global SYMPLICITY Registry. Eur Heart J 2019;doi: 10.1093/eurheartj/ehzI I8.

48. Böhm M, Mahfoud F, Townsend RR, et al. Ambulatory heart rate reduction after catheter-based renal denervation in hypertensive patients not receiving anti-hypertensive medications: Data from SPYRAL HTN-OFF MED, a randomised, sham-controlled, proof-of-concept trial. Eur Heart J 2019; 40:743-751.

49. Fengler K, Rommel K-P, Blazek S, et al. A three-arm randomised trial of different renal denervation devices and techniques in patients with resistant hypertension (RADIOSOUND-HTN). Circulation 2019;139:590-600.

50. Gerstein HC, Colhoun HM, Dagenais GR, et al.; REWIND Investigators. Dulaglutide and cardiovascular outcomes in type 2 diabetes (REWIND): A double-blind, randomised placebo-controlled trial. Lancet 2019; 394:121-130.

51. Wiviott SD, Raz I, Bonaca MP, et al. Dapagliflozin and cardiovascular outcomes in type 2 diabetes. N Engl J Med 2019;380:347-357.

52. Zelniker TA, Wiviott SD, Raz I, et al. SGLT2 inhibitors for primary and secondary prevention of cardiovascular and renal outcomes in type 2 diabetes: A systematic review and meta-analysis of cardiovascular outcome trials. Lancet 2019;393:31-39.

53. Perkovic V, Jardine MJ, Neal B, et al. Canagliflozin and renal outcomes in type 2 diabetes and nephropathy. N Engl J Med 2019;380:2295-2306

54. McMurray JJV, Solomon SD, Inzucchi SE, et al. Dapagliflozin in patients with heart failure and reduced ejection fraction. N Engl J Med 2019; 381:1995-2008.

55. Tardif JC, Kouz S, Waters DD, et al. Efficacy and safety of low-dose colchicine after myocardial infarction. N Engl J Med 2019; 38I (26):2497-2505.

56. Seshasai SR, Wijesuriya S, Sivakumaran R, et al. Effect of aspirin on vascular and nonvascular outcomes: Meta-analysis of randomised controlled trials. Arch Intern Med 2012;172:209-216.

57. Authors/Task Force Members: Piepoli MF, Hoes AW, Agewall S, et al. 2016 European Guidelines on cardiovascular disease prevention in clinical practice: The Sixth Joint Task Force of the European Society of Cardiology and Other Societies on Cardiovascular Disease Prevention in Clinical Practice (constituted by representatives of 10 societies and by invited experts) developed with the special contribution of the European Association for Cardiovascular Prevention \& Rehabilitation (EACPR). Eur Heart J 20 | 6;37:23 I 5-238I. 